Zbl Arbeitsmed 2020 · 70:207-214 https://doi.org/10.1007/s40664-020-00397-4 Online publiziert: 30 . April 2020

(c) Der/die Autor(en) 2020

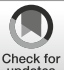

Barbara Hirschwald' · Annette Nold' · Frank Bochmann' · Thomas Heitmann² Yi Sun ${ }^{1}$

'Institut für Arbeitsschutz (IFA), Deutsche Gesetzliche Unfallversicherung (DGUV), Sankt Augustin, Deutschland

${ }^{2}$ Berufsgenossenschaft Holz und Metall (BGHM), Düsseldorf, Deutschland

\title{
Chronotyp, Arbeitszeit und Arbeitssicherheit
}

\author{
Auswirkungen von zirkadianer Rhythmik \\ und Arbeitsbeginn auf die Schlafdauer von \\ Beschäftigten in der holz- und \\ metallverarbeitenden Industrie
}

\section{Fragestellung}

Das moderne Arbeitsleben mit wechselnden Arbeitszeiten, frühem Arbeitsbeginn und Nachtarbeit stört den zirkadianen Rhythmus und die Synchronisation der inneren biologischen Uhr mit dem äußeren Tag-Nacht-Rhythmus. Der physiologische Effekt solcher Arbeitszeiten gleicht dem eines Jetlags: Der Körper befindet sich in einem permanenten Anpassungsstress während einer Arbeitswoche. Schlafstörungen wie Einschlaf- und Durchschlafschwierigkeiten sowie vorzeitiges Aufwachen sind häufig die Folge. Da es Hinweise auf einen $\mathrm{Zu}-$ sammenhang zwischen Schlafstörungen und Arbeitsunfällen gibt [23], wird ein Einfluss des Chronotyps auf das Arbeitsunfallgeschehen vermutet.

Ziel der vorliegenden Arbeit ist die Untersuchung der Einflussfaktoren "Chronotyp“ und „Lage der Arbeitszeit“ auf die Schlafdauer. In • Abb. 1 sind mögliche Wirkungszusammenhänge zwischen der zeitlichen Lage der Arbeit und der Arbeitssicherheit abgebildet [11].

\section{Hintergrund}

\section{Zirkadiane Rhythmik und Chronotyp}

In den sog. Bunkerexperimenten in den sechziger Jahren stellten Aschoff et al. die unterschiedliche Phasenlänge in der Tagesperiodik des Menschen fest. Die Probanden, die für mehrere Wochen unter Ausschluss aller externen Zeitgeber (keine Uhren, kein Tageslicht) lebten, zeigten in ihrem Empfinden für Tag und Nacht nur eine ungefähre Tageslänge (zirkadian), die zwischen 23 und $26 \mathrm{~h}$ lag [2]. Die innere Uhr vieler Menschen geht also schneller oder langsamer als die äußere Uhr. Dass beide Uhren dennoch bei den meisten Menschen übereinstimmen, beruht auf der täglichen Synchronisation der inneren Uhr mit der äußeren Uhr durch den wichtigen Zeitgeber Licht. Die im Auge eintreffende Umgebungshelligkeit wird per Signal an alle Körperzellen weitergeleitet, sodass physiologische Vorgänge reguliert und aufeinander abgestimmt ablaufen können. Die Kopplung des endogenen Rhythmus an äußere Zeitgeber wird als Entrainment bezeichnet und ermöglicht u. a. die Anpassung an eine andere Zeitzone. Ein Jetlag dauert somit nur wenige Tage und lässt sich durch den Aufenthalt im Freien bei Tageslicht noch verkürzen.
Die genetisch festgelegten Phasenlängen der Menschen führten zu einer Klassifizierung in verschiedene Chronotypen. Unterschieden werden im Allgemeinen Frühtypen, Intermediäroder Normaltypen und Spättypen. Die Frühtypen haben eine Phasenlänge von weniger als $24 \mathrm{~h}$ und sind im Verhältnis zur Außenzeit morgens früher fit und abends früher müde als die Spättypen. Letztere haben eine Phasenlänge von mehr als $24 \mathrm{~h}$, sind abends länger wach und können weit in den Vormittag hinein schlafen. Die Intermediärtypen liegen in ihrem zirkadianen Rhythmus zwischen den Früh- und den Spättypen.

Der Chronotyp beeinflusst nicht nur das Schlaf- und Wachverhalten. Typabhängig schwanken auch Stoffwechsel, Organtätigkeit und Konzentrationsfähigkeit innerhalb eines 24-StundenZeitraumes und lassen die zirkadiane Rhythmik eines Individuums erkennen. Melatonin - auch als sog. Schlafhormon bekannt - markiert mit dem abendlichen Anstieg im Körper den Beginn der Nachtphase und erreicht in der Mitte der individuellen Nacht die höchste Konzentration. Gegen Morgen wird die Melatoninproduktion im Körper reduziert und durch natürliches Tageslicht unterdrückt. Eine nächtliche Lichtexposition reduziert die Melatoninausschüttung über die nächsten $24 \mathrm{~h}$. 


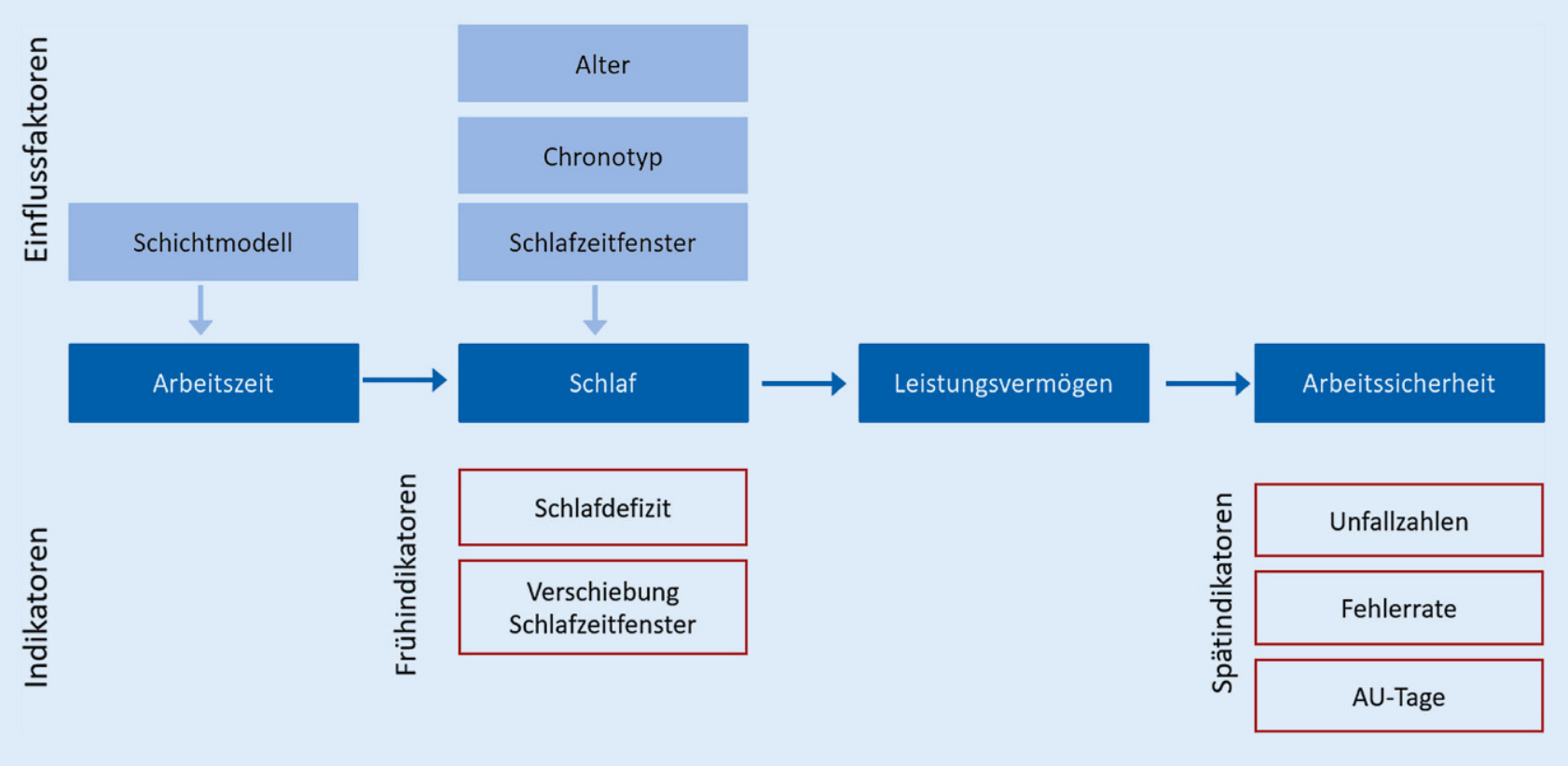

Abb. 1 ॥ Wirkungsmodell zum arbeitszeitbedingten Unfallrisiko. AU Arbeitsunfähigkeit. (Nach Hirschwald et al. [11])

Die Schlafqualität verschlechtert sich. Dem kann tagsüber mit ausreichender Lichtexposition entgegengewirkt werden [10].

\section{Einfluss der zirkadianen Rhythmik auf Konzentration und Leistungsfähigkeit}

Der Medizin-Nobelpreis 2017 wurde für die Entdeckung weiterer Komponenten der inneren Uhr auf genetischer Ebene verliehen [13]. Dies hat auch in der Arbeitswelt die Aufmerksamkeit vermehrt auf die Lage der Arbeitszeit gelenkt und trägt zum Verständnis des Chronotypeinflusses auf die Schichtarbeitstoleranz bei [20]. Im Fahrsimulator machten Probanden beispielsweise mehr Fehler, wenn die Testzeit außerhalb ihrer chronobiologischen Leistungsphasen lag [8]. Ebenso zeigten Flughafenmitarbeiter in Reaktionszeittests erhebliche Konzentrationsschwächen zu Beginn der Frühschicht und am Ende der Nachtschicht [5], also zu Zeiten, in denen der Körper auf Schlaf eingerichtet ist. Nach einer Nachtschicht zeigten weibliche Beschäftigte im Pflegedienst eines Klinikums eine verlängerte mittlere Reaktionszeit und eine höhere Zahl von Auslassungsfehlern im psychomotorischen Vigilanztest [4]. Chellappa et al. zeigten in simulierten Nachtschichten, dass die Aufrechterhaltung der Aufmerksamkeit zum Ende der Nachtschicht hin abnahm und von der davorliegenden Schlafhistorie abhängig war. Außerdem überschätzten die Probanden ihre eigene Leistungsfähigkeit im Vergleich zu den Ergebnissen der anschließend durchgeführten objektiven Tests [7].

\section{Lage der Arbeitszeit als Gefährdungsfaktor}

In $₫ 5$ des Arbeitsschutzgesetzes „Beurteilung der Arbeitsbedingungen“ wird die Arbeitszeit explizit als möglicher Gefährdungsfaktor für Sicherheit und Gesundheit genannt. Rund $10 \%$ der abhängig Beschäftigten in Deutschland haben Arbeitszeiten außerhalb des Zeitfensters zwischen 7:00 und 19:00 Uhr (sog. Normalarbeitszeit); $13 \%$ arbeiten in Wechselschicht, teilweise mit Nachtarbeit bzw. Dauernachtarbeit [3]. Leiden Schichtarbeiter dauerhaft an Tagesmüdigkeit und Schlafstörungen, steigt das Risiko für Erkrankungen und Unfälle. In einer Untersuchung zum Zusammenhang von Schlaf und Arbeitssicherheit fanden Brossoit et al., dass Symptome von Insomnie (Einschlaf- und Durchschlafschwierigkeiten) der stärkste Prädiktor für Sicherheitsrisiken bei der Arbeit sind.
In dieser Studie hatte die Schlafdauer allerdings keinen Einfluss auf die Arbeitssicherheit, da die Probanden durchschnittlich $7 \pm 1 \mathrm{~h}$ schliefen [6]. Ein systematisches Review mit Metaanalyse zeigte, dass etwa $13 \%$ der Arbeitsunfälle durch Müdigkeit verursacht werden [23]. Die vorliegende Studie soll einen Beitrag zur Ursachenerforschung und Vermeidung arbeitszeitbedingter Unfälle leisten.

\section{Methode}

In einem Kooperationsprojekt zwischen dem Institut für Arbeitsschutz (IFA) und der Berufsgenossenschaft Holz und Metall (BGHM) wurden Beschäftigte mit meldepflichtigem Arbeitsunfall aus Mitgliedsbetrieben der BGHM kontaktiert. Die Befragungen mittels Fragebögen erfolgten retrospektiv im Rahmen von stichprobenartigen Nachuntersuchungen meldepflichtiger Arbeitsunfälle durch die Präventionsfachkräfte/ Aufsichtspersonen. Der zeitliche Abstand zwischen dem Arbeitsunfall und der Befragung betrug im Allgemeinen nicht mehr als 2 Wochen, bei schweren Unfällen auch länger. Der genaue Befragungszeitpunkt wurde bei dieser Erhebung nicht erfasst. Die Arbeitsunfälle hatten sich zwischen 2013 und 2018 ereignet. Da die gesetzliche Un- 
Zbl Arbeitsmed 2020 · 70:207-214 https://doi.org/10.1007/s40664-020-00397-4

(c) Der/die Autor(en) 2020

B. Hirschwald · A. Nold · F. Bochmann · T. Heitmann · Y. Sun

\section{Chronotyp, Arbeitszeit und Arbeitssicherheit. Auswirkungen von zirkadianer Rhythmik und Arbeitsbeginn auf die Schlafdauer von Beschäftigten in der holz- und metallverarbeitenden Industrie}

\section{Zusammenfassung}

Hintergrund und Zielsetzung. Viele Arbeitszeitregelungen greifen in den Biorhythmus der Beschäftigten ein und wirken sich negativ auf Konzentration und Schlafqualität aus. Schichtarbeitende leiden doppelt so häufig an Schlafstörungen wie andere Erwerbstätige. Schlafmangel und gestörter Schlaf erhöhen das Unfallrisiko. In dieser Studie wurde untersucht, ob die Schlafdauer von der Lage der Arbeitszeit und vom individuellen Chronotyp beeinflusst wird.

Methode. In einer Querschnittsuntersuchung wurden 374 Beschäftigte mit meldepflichtigem Arbeitsunfall zu ihrem Chronotyp und ihren üblichen Schlafenszeiten befragt. Zur Chronotypermittlung wurde die
Composite Scale of Morningness (CSM) in der deutschsprachigen Version verwendet. Die Effekte von Schichtarbeit, Chronotyp und Alter auf die Schlafdauer wurden mittels multivariabler linearer bzw. logistischer Regression untersucht. Ergebnisse. Ohne Schichtarbeit (Tagarbeitszeiten [TA]) betrug die durchschnittliche Schlafdauer an Arbeitstagen 7,2 h (SD 0,9). Vor einer Frühschicht (FS) war die Schlafdauer verkürzt auf $6,4 \mathrm{~h}(\mathrm{SD} 0,6)$. Ein früher Arbeitsbeginn ist ein Risikofaktor für eine kurze Schlafdauer $(<7 \mathrm{~h})$ vor FS Odds Ratio $(\mathrm{OR})=13,8(95 \%$ Konfidenzintervall [KI] 3,38-56,25) im Vergleich zu einem späten Arbeitsbeginn (Spätschicht). Ein später
Chronotyp erhöht ebenfalls das Risiko für kurze Schlafdauer an Arbeitstagen $\mathrm{OR}=2,7$ (95\% Kl 0,97-7,67). An arbeitsfreien Tagen haben Frühtypen eine signifikant kürzere Schlafdauer als Intermediärtypen $\mathrm{OR}=2,2$ (95\% KI 1,16-4,31).

Diskussion. Ein späterer und flexibler Arbeitsbeginn unter Einbeziehung des Chronotyps sowie Maßnahmen für gesundheitsförderlichen Schlaf könnten dazu beitragen, müdigkeitsbedingte Arbeitsunfälle zu verhindern.

\section{Schlüsselwörter}

Schlafmangel · Schichtarbeit · Arbeitsunfälle · Prävention · Müdigkeit

\section{Chronotype, working time and occupational safety. The impact of circadian rhythm and start of working time on sleep duration of employees in the wood and metalworking industry}

\begin{abstract}
Background and objective. Atypical work times, such as night shifts and very early shifts can have a negative effect on sleep quality and concentration of employees. Sleep disorders are twice as frequent in shift workers as in other workers. Short sleep duration and disturbed sleep are associated with an increased risk of accidents. The purpose of this study was to investigate whether the start of working time and the individual chronotype affect sleep duration.

Methods. In a cross-sectional study, 374 employees with a reportable accident at work were asked about their chronotype and normal sleep routine. The employees were chronotyped using the 13-item composite scale of morningness (CSM) questionnaire in
\end{abstract}

the German version. The middle point of sleep was calculated from sleep onset and time of waking up on free days. The effects of shift work, chronotype and age on sleep duration were assessed with multivariable linear or logistic regression.

Results. The mean sleep duration without shift work (day work, DW) was 7.2 h (SD $0.9 \mathrm{~h}$ ). Working early shifts (ES) shortened the sleep duration to $6.4 \mathrm{~h}$ (SD $0.6 \mathrm{~h}$ ). An early start to work is a risk factor for short sleep duration $(<7 \mathrm{~h})$ before $\mathrm{ES}$ odds ratio, $\mathrm{OR}=13.8(95 \%$ confidence interval, $\mathrm{Cl}$ 3.38-56.25), compared to a late start of working time (evening shift). Being an evening type is also a risk factor for short sleep duration on working days $\mathrm{OR}=2.7$ (95\% Cl 0.97-7.67). On days off morning types have a significantly shorter sleep duration than intermediate types $\mathrm{OR}=2.2(95 \% \mathrm{Cl}$ 1.16-4.31).

Discussion. A later and more flexible start to work adapted to the chronotype could attenuate the sleep deficit on working days. Working hours that enable a sufficiently long sleeping time and information on healthpromoting sleep could contribute to the prevention of fatigue-related accidents at work.

\section{Keywords}

Sleep deprivation · Shift work · Occupational accidents · Prevention · Sleepiness fallversicherung kaum Einfluss auf die Verkehrssicherheit von Arbeitnehmern hat, werden Wegeunfälle üblicherweise nicht nachuntersucht. Personen mit Wegeunfall wurden daher nicht befragt. Die Teilnahme war freiwillig, und alle Angaben wurden anonymisiert. Fragebögen mit widersprüchlichen Datenangaben, wie z. B. der Überschneidung von Schlafzeit und Arbeitszeit, wurden von der Auswertung ausgeschlossen.
Befragung zu Chronotyp, Schlafund Arbeitszeiten

Der Composite-Scale-of-MorningnessFragebogen (CSM) in der deutschen Fassung [16, 21] ist ein validiertes Instrument zur Erhebung des Chronotyps. Die Reliabilität wurde in Form von Cronbachs Alpha berechnet und liegt für drei verschiedene deutsche Stichproben zwischen 0,84 und 0,87. Zur Validität wurden Korrelationen zu verschiedenen physiologischen und psychologischen Parametern sowie zur deutschsprachigen Version des Morningness-EveningnessFragebogens [12] berechnet. Es zeigten sich signifikante Zusammenhänge zwischen dem CSM-Wert und dem SchlafWach-Rhythmus, aber nicht zwischen dem CSM-Wert und der Schlafdauer. Auch konnten signifikante Zusammenhänge zu physiologischen und kognitiven Leistungsspitzen nachgewiesen werden [15]. Um die vorliegende $\mathrm{Zu}$ - 


\begin{tabular}{|c|c|c|c|c|c|c|}
\hline CSM-Punkte & $55-50$ & 49-44 & $43-38$ & $37-32$ & $31-26$ & $25-13$ \\
\hline Chronotypkategorie & 1 & 2 & 3 & 4 & 5 & 6 \\
\hline Chronotyp & & typ & & ediärtyp & & ättyp \\
\hline Schlafmitte ${ }^{a}$ & $0: 00-2: 00$ & $2: 00-3: 00$ & $3: 00-4: 00$ & $4: 00-5: 00$ & $5: 00-6: 00$ & $6: 00-12: 00$ \\
\hline
\end{tabular}

Tab. 2 Beschreibung der Stichprobe N (\%)

\begin{tabular}{l|c}
\hline $\begin{array}{l}\text { Gesamtanzahl } N \\
\text { davon männlich }\end{array}$ & 374 \\
\hline Alter in Jahren & $358(95,7)$ \\
\hline$\leq 30$ & $123(33,0)$ \\
\hline $31-50$ & $137(36,7)$ \\
\hline$>50$ & $113(30,3)$ \\
\hline Vollständigkeit der Angabe & $373(99,7)$ \\
\hline Arbeitszeit (Lage) & \\
\hline Frühschicht & $75(20,1)$ \\
\hline Spätschicht & $33(8,8)$ \\
\hline Nachtschicht & $24(6,4)$ \\
\hline Tagarbeit & $242(64,7)$ \\
\hline Schlafdauer an Arbeitsta- & $7,1 \pm 1,0^{\mathrm{a}}$ \\
\hline gen (h) & \\
\hline$\leq 6,0$ & $53(16,6)$ \\
\hline $6,1-7,0$ & $125(39,2)$ \\
\hline $7,1-8,0$ & $103(32,3)$ \\
\hline$>8,0$ & $38(11,9)$ \\
\hline Vollständigkeit der Angabe & $319(85,3)$ \\
\hline $\begin{array}{l}\text { Schlafdauer an freien Ta- } \\
\text { gen (h) }\end{array}$ & $8,1 \pm 1,3^{\mathrm{a}}$ \\
\hline$\leq 6,0$ & $21(6,3)$ \\
\hline $6,1-7,0$ & $55(16,5)$ \\
\hline $7,1-8,0$ & $102(30,6)$ \\
\hline$>8,0$ & $155(46,6)$ \\
\hline $\begin{array}{l}\text { Vollständigkeit der Angabe } \\
\text { Chronotyp (CSM-Punkte) }\end{array}$ & $333(89,0)$ \\
\hline Frühtyp (55-44) & $41,4 \pm 6,0^{\mathrm{a}}$ \\
\hline $\begin{array}{l}\text { Intermediärtyp (43-32) } \\
\text { Spättyp (31-13) }\end{array}$ & $152(40,6)$ \\
\hline ä Mittelwert und einfache Standardabwei- \\
chung & $197(52,7)$ \\
\hline & $25(6,7)$ \\
\hline
\end{tabular}

fallsstichprobe bezüglich des Chronotyps einzuschätzen, wurde sie mit anderen Kollektiven aus der Literatur verglichen [17].

Der CSM-Fragebogen umfasst 13 Fragen mit 4 oder 5 unterschiedlichen Antwortmöglichkeiten. Durch die hypothetische Fragestellung wie z.B. „Wann würden Sie aufstehen/zu Bett gehen?“ oder „Wann würden Sie eine körperlich/ geistig anstrengende Tätigkeit terminieren, wenn es nur nach Ihrem Wohlbefinden ginge?" werden die individuell bevorzugten Zeiten für Schlafen und Aktivitäten erfasst. Außerdem schätzen die Befragten ihre tatsächliche Wachheit früh morgens (6:00) bzw. ihre Müdigkeit abends ein. Das Ergebnis ist ein Wert zwischen 55 Punkten für extreme Frühtypen und 13 Punkten für extreme Spättypen (CSM-Wert), wobei Chronotyp als Kontinuum zu verstehen ist. Für die grafischen Darstellungen wurden die CSM-Punkte in 6 Chronotypkategorien zusammengefasst (• Tab. 1). Die ursprünglich geplante 7. Kategorie (13-19 Punkte) enthält nur 2 Datensätze und wurde daher in die 6. Kategorie einbezogen. Für einige Auswertungen wurden die 6 Chronotypkategorien noch weiter zusammengefasst, um eine statistisch auswertbare Anzahl an Datensätzen pro Gruppe zu erhalten. Kategorie 1 und 2 werden als Frühtypen bezeichnet, Kategorie 3 und 4 als Intermediärtypen und Kategorie 5 und 6 als Spättypen.

Für die Studie wurden ergänzend zu den 13 Fragen des standardisierten CSM-Fragebogens die Aufsteh- und Zubettgehzeiten sowie die Einschlaf-und Aufwachzeiten an Arbeitstagen und an arbeitsfreien Tagen erfragt. Die Teilnehmer wurden gebeten, die üblichen Einschlaf- und Aufwachzeiten in Bezug auf die Arbeitszeit am Unfalltag anzugeben. Aus den Einschlaf- und Aufwachzeiten an freien Tagen wurde die Schlafmitte berechnet. Dies ist auf einer Zeitachse der Mittelwert von Einschlaf- und Aufwachzeit. Eine Korrektur der errechneten Schlafmitte um ein eventuelles arbeitstägliches Schlafdefizit konnte nicht durchgeführt werden, da die Schlafhistorie nicht erfasst wurde. Die umgekehrt proportionale Zuordnung von CSM-Punkten zu Chro- notypkategorien wurde gewählt, damit die kleinste Chronotypkategorie der frühesten Schlafmitte entspricht (• Tab. 1). Während die Schlafmitte ein Maß für die individuell bevorzugte Schlafenszeit ist, misst der CSM-Wert auch den Schlafund Aktivitätsrhythmus. Dieser lässt auch Rückschlüsse auf den zeitlichen Ablauf physiologischer Prozesse im Körper zu. Sowohl die Schlafmitte als auch der CSM-Wert sind unabhängig von der Schlafdauer.

Alter, Geschlecht, Arbeitszeit am Unfalltag und Schichtarbeit (ja/nein) wurden ebenfalls erhoben. Die Frühschichten begannen zwischen 5:00 und 6:30 Uhr, für Spätschichten lag der Arbeitsbeginn zwischen 12:30 und 14:30 Uhr und die Nachtschicht begann zwischen 20:00 und 22:00. Die Schichtdauer betrug 7,5-8,5h. Personen, die Schichtarbeit verneint hatten, wurden als Tagarbeiter bezeichnet, unabhängig davon, ob sie flexible oder feste Arbeitszeiten hatten.

\section{Statistische Auswertung}

Die Auswertung der Ergebnisse erfolgte zunächst deskriptiv unter Angabe des arithmetischen Mittel (M) und der Standardabweichung (SD). Zur Ermittlung des Signifikanzniveaus $(p<0,05)$ wurde der Mann-Whitney-U-Test gerechnet, da die CSM-Werte nicht normalverteilt waren (-Abb. 2). Mit multiplen linearen bzw. logistischen Regressionen wurde der Einfluss der Variablen „Chronotyp“, „Arbeitsbeginn“ und „Alter“ auf die Schlafdauer an Arbeitstagen und an freien Tagen untersucht. Personen mit einem Unfall während der Nachtschicht hatten teilweise keine Schlafdaten oder nichtplausible Aufstehzeiten angegeben, die weniger als $4 \mathrm{~h}$ nach dem Arbeitsende lagen. Daher wurde die Nachtschicht von den Regressionsanalysen ausgeschlossen. Al- 


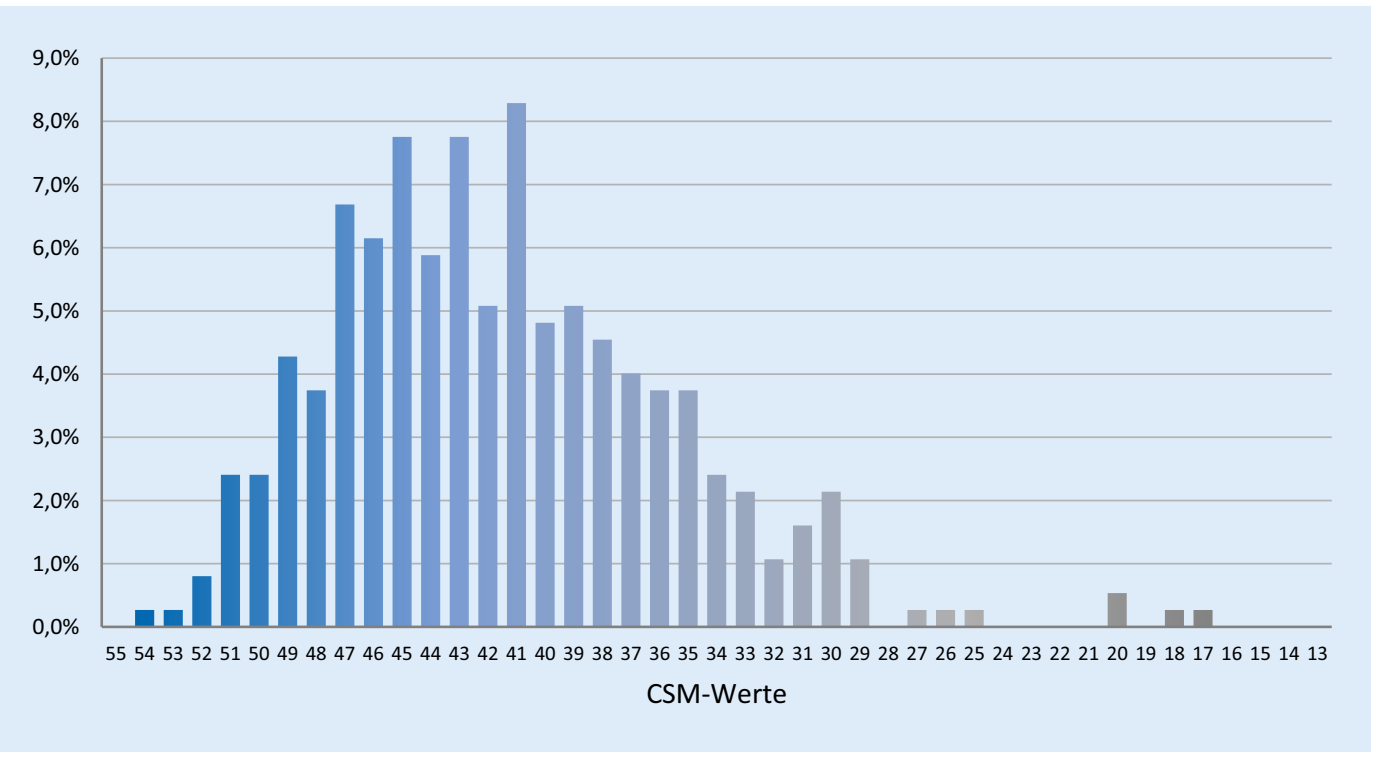

Abb. $2 \triangleleft$ Prozentuale Verteilung der CSM-Werte (absteigende Skalierung). CSM "Composite Scale of Morningness"

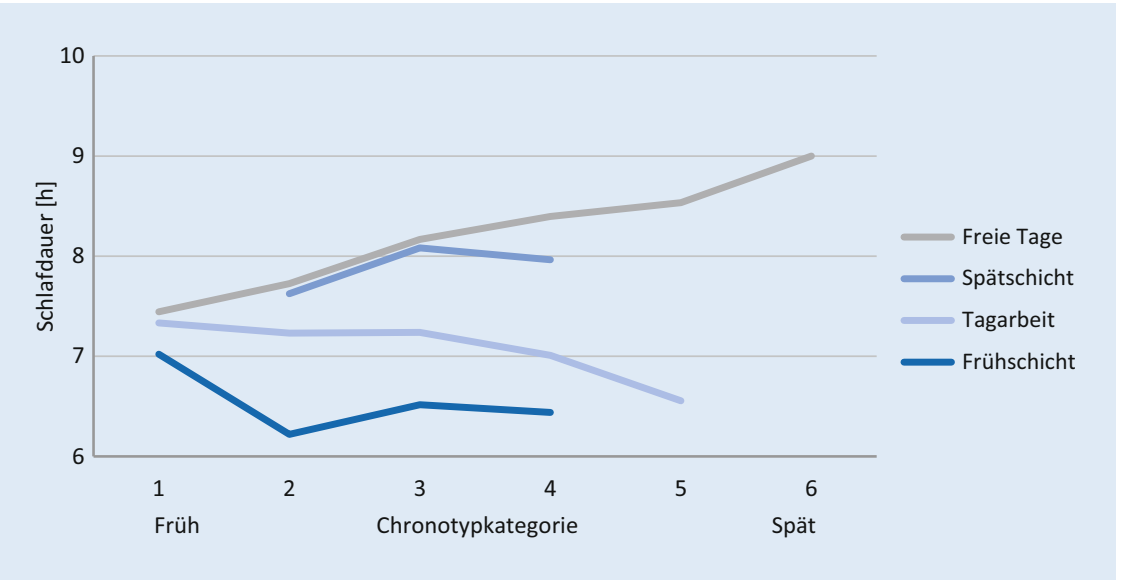

Abb. 3 ॥ Schlafdauer in Stunden vor Arbeitstagen und an freien Tagen in Abhängigkeit vom Chronotyp

le Analysen wurden mit dem Softwarepaket SAS 9.4 (SAS Institute GmbH, Heidelberg, Deutschland) durchgeführt.

\section{Ergebnisse}

\section{Studienpopulation}

Die Daten von 374 Personen mit einem meldepflichtigen Arbeitsunfall konnten in die Auswertung eingeschlossen werden. Das Durchschnittsalter lag bei $39,7 \pm 14,1$ Jahren; $96 \%$ der Befragten waren männlich. Im befragten Kollektiv beantworteten $35 \%$ der Teilnehmer die Frage nach Schichtarbeit mit "Ja“. Eine Unterscheidung zwischen Wechselschicht und Dauerschicht konnte aus dieser Antwort nicht abgeleitet wer- den. Die Ergebnisse der Befragung zeigt - Tab. 2.

Insgesamt $20 \%$ der Befragten erlitten den Arbeitsunfall während einer Frühund $6 \%$ hatten in der Nachtschicht gearbeitet. Die verbleibenden $65 \%$ hatten die Frage nach Schichtarbeit verneint. Eine kurze Schlafdauer mit maximal $7 \mathrm{~h}$ gaben $55,8 \%$ der Befragten an, $12 \%$ schliefen an Arbeitstagen länger als $8 \mathrm{~h}$. An arbeitsfreien Tagen vergrößerte sich der Anteil derer, die mehr als $8 \mathrm{~h}$ schliefen auf $47 \%$, während nur $23 \%$ eine kurze Schlafdauer mit höchstens $7 \mathrm{~h}$ angaben. Die Klassifizierung in Chronotypen auf Basis des CSM-Werts zeigt, dass im befragten Kollektiv ca. 6-mal so viele Frühtypen wie Spättypen vorkamen (41\% vs. $7 \%$ ). $53 \%$ schicht, 9\% während einer Spätschicht, der Befragten gehören zu den Intermediärtypen.

\section{Korrelation von CSM-Wert und Schlafmitte}

An arbeitsfreien Tagen zeigen die Schlafmitten chronotypspezifische Unterschiede. Die Schlafmitten ausgeprägter Frühtypen (2:24 Uhr) und ausgeprägter Spättypen (6:30 Uhr) weisen eine Differenz von ca. $4 \mathrm{~h}$ auf (• Tab. 3). Der SpearmanKorrelationskoeffizient zwischen CSMWert und Schlafmitte beträgt $r=-0,631$ (95\% Konfidenzintervall [KI] $-0,695$ bis $-0,557)$.

\section{Häufigkeitsverteilung des Chronotyps}

In $\bullet$ Abb. 2 wird die relative Häufigkeit des CSM-Wertes in Prozent gezeigt. Die linksschiefe Verteilung der Werte bedeutet, dass die hier befragten Personen verglichen mit einer Normalverteilung häufiger höhere CSM-Werte besaßen, also häufiger einen früheren Chronotyp aufwiesen.

\section{Chronotyp, zeitliche Lage der Arbeit und Schlafdauer}

Die Schlafdauer an Arbeitstagen ist vor der Frühschicht für alle Chronotypgruppen am kürzesten, gefolgt von Tagarbeit und Spätschicht (• Abb. 3). In der Stich- 


\begin{tabular}{|c|c|c|c|c|c|c|}
\hline Chronotypkategorie & 1 & 2 & 3 & 4 & 5 & 6 \\
\hline CSM-Punkte & $55-50$ & $49-44$ & $43-38$ & $37-32$ & $31-26$ & $25-13$ \\
\hline \multirow[t]{2}{*}{ Schlafmitte (SD) ${ }^{\mathrm{a}}$} & $2: 24(48)$ & $3: 12(51)$ & 4:03 (66) & $4: 42(60)$ & $5: 06(54)$ & $6: 30(126)$ \\
\hline & \multicolumn{2}{|c|}{$3: 06(54)$} & \multicolumn{2}{|c|}{$4: 18(66)$} & \multicolumn{2}{|c|}{$5: 18(72)$} \\
\hline Chronotyp & \multicolumn{2}{|l|}{ Frühtyp } & \multicolumn{2}{|c|}{ Intermediärtyp } & \multicolumn{2}{|l|}{ Spättyp } \\
\hline
\end{tabular}

probe befanden sich nur wenige Personen mit ausgeprägt spätem Chronotyp (Kategorie 6), sodass für die Darstellung der Schlafdauer an Arbeitstagen für diese Gruppe zu wenig auswertbare Daten vorliegen. Das Gleiche gilt für Frühund Spättypen (Chronotypkategorie 1, 5 und 6) in Spätschicht. Für Personen mit Nachtschichtarbeit liegen insgesamt zu wenig Schlafdaten für eine statistische Auswertung nach Chronotyp vor.

Vor einer Frühschicht betrug die durchschnittliche Schlafdauer in der Stichprobe $6,4 \pm 0,6 \mathrm{~h}$, wobei ausgeprägte Frühtypen $30 \mathrm{~min}$ länger schliefen als alle anderen Chronotypen. Bei Tagarbeit schliefen Spättypen 42 min weniger als alle anderen Chronotypen.

An arbeitsfreien Tagen schliefen Frühtypen durchschnittlich $1,3 \mathrm{~h}$ weniger als Spättypen $(7,7 \pm 1,3 \mathrm{~h}$ vs. $9,0 \pm 1,4 \mathrm{~h})$. Je später der Chronotyp, umso länger war die Schlafdauer.

\section{Einflussfaktoren auf die Schlafdauer}

In einer multiplen linearen Regressionsanalyse wurde der Einfluss folgender Faktoren auf die Schlafdauer an Arbeitstagen untersucht:

- Arbeitsbeginn: Frühschicht und Tagarbeit im Vergleich zur Spätschicht,

- Chronotyp: Frühtyp und Spättyp im Vergleich zum Intermediärtyp,

- Alter als kontinuierliche Variable.

Der Arbeitsbeginn zeigt einen signifikanten Einfluss auf die Schlafdauer an Arbeitstagen (-Tab.4). Bei Frühschichtarbeit, aber auch bei Tagarbeit ist die Schlafdauer gegenüber der Spätschicht verkürzt. Die Spätschicht wurde als Referenz gewählt, weil man davon ausgehen kann, dass die Schlafphase nicht durch den Arbeitsbeginn verkürzt wird. Au- ßerdem zeigt auch ein später Chronotyp einen signifikanten Einfluss auf die Schlafdauer an Arbeitstagen. Das Alter zeigt hier keinen Einfluss.

\section{Risikofaktoren für verkürzte Schlafdauer an Arbeitstagen und freien Tagen}

Um herauszufinden, welche Faktoren die Schlafdauer an Arbeitstagen verkürzen können, wurden die Daten von Personen mit einer Schlafdauer von weniger als $7 \mathrm{~h}$ gesondert analysiert; $7 \mathrm{~h}$ Schlaf entsprach dem Median der Schlafdauer an Arbeitstagen. Die Ergebnisse sind in - Tab. 5 dargestellt.

Die Frühschicht mit einem Arbeitsbeginn um 6:00 Uhr erhöhte das Risiko, weniger als $7 \mathrm{~h}$ zu schlafen, um das ca. 14-fache (OR=13,8). Unabhängig davon trug auch ein später Chronotyp zur Erhöhung des Risikos bei $(\mathrm{OR}=2,7)$. Das Alter hatte hier keinen Einfluss auf die Schlafdauer. Berechnet man die Risikofaktoren für kurze Schlafdauer an freien Tagen, so hat die Arbeitszeit erwartungsgemäß keinen Einfluss mehr. Allerdings erhöht nun ein früher Chronotyp das Risiko für eine verkürzte Schlafdauer an freien Tagen. Das Alter bleibt ebenfalls ohne Einfluss. Eine Subanalyse für Männer zeigte keinen Einfluss des Geschlechts auf die Studienergebnisse in dieser Population. Aufgrund der relativ kleinen Stichprobengröße von Frühschichtarbeitenden $(n=75)$ sowie Spätschichtarbeitenden $(n=33)$ ist das Konfidenzintervall des Effektschätzers von Frühschicht im Regressionsmodell vergleichsweise groß.

\section{Diskussion}

In den meisten Studien zu den Auswirkungen von Schichtarbeit aufGesundheit und Arbeitssicherheit liegt der Fokus auf der Nachtarbeit. Die Frühschicht wird bei Vergleichen von Schichtsystemen häufig als Referenz im Sinne einer Arbeitszeit ohne negative Auswirkungen angesehen. Die hier vorliegenden Ergebnisse belegen allerdings, dass ein Arbeitsbeginn um 6:00 der stärkste Prädiktor für eine verkürzte Schlafdauer ist. Die Betrachtung von Einschlaf- und Aufwachzeiten ließ erkennen, dass der frühe Arbeitsbeginn zur Kappung des Schlafes am Morgen führte. Das frühe Aufstehen wird nicht durch entsprechend früheres $\mathrm{Zu}$ Bett-Gehen kompensiert. Eine skandinavische Studie zeigt, dass sich die Schlafdauer für jede Stunde, die die Arbeit vor 8:00 Uhr beginnt, um $35 \mathrm{~min}$ reduziert [1].

Stothard et al. verglichen die Schlafzeiten und die Melatoninspiegel derselben Personen im Sommer und im Winter unter 2 Bedingungen: überwiegend künstliche Beleuchtung (übliche Alltags- und Arbeitsbedingungen) und ausschließlich Tageslicht und natürliche Dunkelheit (Campingaufenthalt). Die zirkadiane Phasenlage unter Alltagsund Arbeitsbedingungen lag im Sommer um 1,5 h und im Winter sogar um 2,5h später als beim Campingaufenthalt. Grund hierfür waren die späteren Einschlafzeiten der Probanden unter Alltags-und Arbeitsbedingungen [22]. Die Kombination aus späterer zirkadianer Phasenlage und frühem Arbeitsbeginn im Alltag könnte zwei negative Effekte haben: Erstens, frühes Zu-Bett-Gehen wird erschwert, da der abendliche Melatoninanstieg verspätet einsetzt. Zweitens, bei frühem Arbeitsbeginn wird der Schlaf morgens vorzeitig beendet. Die Folge ist eine Verkürzung der Schlafdauer sozusagen an beiden Enden.

An arbeitsfreien Tagen gaben $47 \%$ der Befragten an, länger als $8 \mathrm{~h} \mathrm{zu}$ schlafen. Diese langen Schlafdauern lassen 
Tab. 4 Einflussfaktoren auf die Schlafdauer

\begin{tabular}{l|l|l}
\hline Variable & \multicolumn{1}{l}{$\boldsymbol{\beta}$} & $\boldsymbol{p}$ \\
\hline Arbeitsbeginn/Schichttyp & \\
\hline Spätschicht & 0 (Referenz) & - \\
\hline Tagarbeit & $-0,62^{*}$ & $0,004^{*}$ \\
\hline Frühschicht & $-1,38^{*}$ & $<0,0001^{*}$ \\
\hline Chronotyp & & \\
\hline Intermediärtyp & 0 (Referenz) & - \\
\hline Frühtyp & $-0,02$ & 0,825 \\
\hline Spättyp & $-0,71^{*}$ & $<0,001^{*}$ \\
\hline Alter & 0,003 & 0,370 \\
\hline *Statistisch signifikant & \\
\hline
\end{tabular}

vermuten, dass ein kumuliertes Schlafdefizit aus der Arbeitswoche an freien Tagen ausgeglichen werden soll. Allerdings trägt der lange Erholungsschlaf an freien Tagen nicht dazu bei, den inneren Rhythmus an den natürlichen TagNacht-Rhythmus anzugleichen [9]. Im Gegenteil: Durch spätes Aufstehen verschiebt sich die Einschlafzeit in den späten Abend oder die Nacht und vergrößert dadurch die Abweichung von innerer und äußerer Uhr. Möglicherweise ist frühes Einschlafen nach freien Tagen erschwert. Die kognitiven Auswirkungen eines chronischen Schlafdefizits werden von den Betroffenen häufig nicht wahrgenommen [14, 24] und können daher ein relevanter Faktor in Bezug auf die Arbeitssicherheit sein.

Im befragten Kollektiv von Personen mit Arbeitsunfall erlebten sowohl frühe als auch späte Chronotypen Schlafzeitverkürzungen $(<7 \mathrm{~h})$, allerdings unter verschiedenen Bedingungen. Spättypen hatten ein erhöhtes Kurzschlaf-Risiko an Arbeitstagen $(\mathrm{OR}=2,7)$, während Frühtypen ein erhöhtes Kurzschlaf-Risiko an freien Tagen zeigten $(\mathrm{OR}=2,2)$. Die intermediären Chronotypen schliefen an Arbeitstagen ähnlich viel wie Frühtypen und konnten zusätzlich durch längeres Ausschlafen an freien Tagen Schlaf nachholen. Im vorliegenden Kollektiv von Erwerbspersonen mit Arbeitsunfall ist die linksschiefe Verteilung des Chronotyps auffällig, d.h. Frühtypen sind überproportional häufig vertreten. Im Vergleich zu einem von Randler et al. befragten Kollektiv aus Deutschland erreichten die hier befragten Personen in allen 4 Altersgrup-

\begin{tabular}{lll} 
Tab. 5 & Risikofaktoren für verkürzte Schlafdauer $(<7 \mathrm{~h})$ & \\
& An Arbeitstagen & An freien Tagen \\
\hline Variable & Odds-Ratio (95\% Konfidenzintervall) & Odds-Ratio (95\% Konfidenzintervall) \\
$\begin{array}{l}\text { Chronotyp } \\
\text { Intermediärtyp }\end{array}$ & 1 (Referenz) & 1 (Referenz) \\
Frühtyp & $1,16(0,68-1,95)$ & $2,23^{*}(1,16-4,31)$ \\
Spättyp & $2,73(0,97-7,67)$ & $0,45(0,06-3,58)$ \\
Arbeitsbeginn/Schichttyp & \\
Spätschicht & 1 (Referenz) & $1($ Referenz) \\
\hline Tagarbeit & $2,92(0,78-10,89)$ & $1,54(0,43-5,47)$ \\
Frühschicht & $13,79 *(3,38-56,25)$ & $1,11(0,27-4,57)$ \\
Alter & $0,99(0,98-1,02)$ & $1,01(0,99-1,03)$ \\
\hline *Statistisch signifikant &
\end{tabular}

pen $(18-20 ; 21-30 ; 31-50$; $\geq 51$ Jahre ) höhere CSM-Werte [17]. Ein altersbedingter Grund für die linksschiefe Verteilung der CSM-Werte kann daher ausgeschlossen werden.

Saksvik et al. erhalten in ihrer Metaanalyse heterogene Ergebnisse mit einer leichten Tendenz zu einer schlechteren Schichtarbeitsverträglichkeit für Frühtypen [20]. Die Formel „Frühtyp in die Frühschicht“ und „Spättyp in die Spätbzw. Nachtschicht" scheint zumindest in Bezug auf die Schlafdauer zu einfach. Möglicherweise können Spättypen sich besser, eventuell auch dauerhafter an wechselnde Schlafenszeiten anpassen, während Frühtypen bei der Lage ihrer Schlafenszeit weniger flexibel sind.

Die vorliegende Befragung liefert einen Beitrag zur Interpretation der Faktoren, die den Schlaf von Beschäftigten beeinflussen. Die retrospektive Erhebung sollte durch die Formulierung der Frage nach den üblichen Schlafzeiten nicht durch mangelnde Erinnerung verzerrt sein. Die Chronotypbestimmung mittels eines validierten Instrumentes zeigt die reale Verteilung der Chronotypen in einem Kollektiv von Erwerbstätigen. Zusammenhänge von Chronotyp, Arbeitszeit und Schlafdauer sind in den statistischen Analysen erkennbar. Die Schlafqualität sowie jahreszeitliche Unterschiede in der Schlafdauer wurden in dieser Studie nicht berücksichtigt.

Für die zukünftige Forschung könnte eine Erfassung von Schlafparametern am Unfalltag sowie an den Tagen davor wertvolle Erkenntnisse liefern. Eine Abfrage von Schlafstörungen würde eine sinnvol- le Ergänzung darstellen. Damit könnten Ursachenanalysen durchgeführt werden, die in dieser Studie noch nicht möglich waren. In Anbetracht von vermutet ca. $13 \%$ müdigkeitsbedingten Arbeitsunfällen scheint die Einbeziehung der Schlafgesundheit in die Unfallerforschung und -vermeidung angezeigt.

\section{Fazit für die Praxis}

- Eine Veränderung in der Arbeitsorganisation mit einem späteren Arbeitsbeginn insbesondere der Frühschicht könnte zur Verlängerung der Schlafdauer beitragen.

- Jüngere und ältere Personen haben verschiedene Risikofaktoren, da mit zunehmendem Alter der zirkadiane Rhythmus früher wird und die Schlafqualität abnimmt. Durch die Berücksichtigung des Chronotyps in der Schichtplanung und -zuteilung könnte die Schlafqualität verbessert werden.

- Ein Aufenthalt im Freien bei Tageslicht sollte auch an Arbeitstagen ermöglicht werden, um die zirkadiane Rhythmik zu stärken und einen früheren abendlichen Melatoninanstieg zu fördern. Der Kreislauf von Schlaf-Phasen-Verschiebung und zu kurzem Schlaf in Folge sollte verhindert werden.

- Im Rahmen der arbeitsmedizinischen Untersuchung sollten Schlafstörungen thematisiert werden (Insomniescreening) und Beratungen und Schlaftrainings angeboten werden. 


\section{- Allen Mitarbeitern sollten Informa- tionen zur Schlafgesundheit zur Verfügung gestellt werden.}

\section{Korrespondenzadresse}

\section{Dipl.-Biol. Barbara Hirschwald}

Institut für Arbeitsschutz (IFA), Deutsche Gesetzliche Unfallversicherung (DGUV)

Alte Heerstr. 111, 53757 Sankt Augustin, Deutschland

barbara.hirschwald@dguv.de

Funding. Open Access funding provided by Projekt DEAL.

\section{Einhaltung ethischer Richtlinien}

Interessenkonflikt. B. Hirschwald, A. Nold, F. Bochmann, T. Heitmann und $Y$. Sun geben an, dass kein Interessenkonflikt besteht.

Für diesen Beitrag wurden von den Autoren keine Studien an Menschen oder Tieren durchgeführt. Für die aufgeführten Studien gelten die jeweils dort angegebenen ethischen Richtlinien.

Open Access Dieser Artikel wird unter der Creative Commons Namensnennung 4.0 International Lizenz veröffentlicht, welche die Nutzung, Vervielfältigung, Bearbeitung, Verbreitung und Wiedergabe in jeglichem Medium und Format erlaubt, sofern Sie den/die ursprünglichen Autor(en) und die Quelle ordnungsgemäß nennen, einen Link zur Creative Commons Lizenz beifügen und angeben, ob Änderungen vorgenommen wurden.

Die in diesem Artikel enthaltenen Bilder und sonstiges Drittmaterial unterliegen ebenfalls der genannten Creative Commons Lizenz, sofern sich aus der Abbildungslegende nichts anderes ergibt. Sofern das betreffende Material nicht unter der genannten Creative Commons Lizenz steht und die betreffende Handlung nicht nach gesetzlichen Vorschriften erlaubt ist, ist für die oben aufgeführten Weiterverwendungen des $\mathrm{Ma}$ terials die Einwilligung des jeweiligen Rechteinhabers einzuholen.

Weitere Details zur Lizenz entnehmen Sie bitte der Lizenzinformation auf http://creativecommons.org/ licenses/by/4.0/deed.de.

\section{Literatur}

1. Akerstedt T, Kecklund G, Selen J (2010) Early morning work - prevalence and relation to sleep/ wake problems: a national representative survey. Chronobiol Int 27:975-986

2. Aschoff J, Wever R (1962) Spontanperiodik des Menschen bei Ausschluß aller Zeitgeber. Naturwissenschaften 49:337-342

3. Backhaus N, Tisch A, Wöhrmann AM (2018) BAuAArbeitszeitbefragung: Vergleich 2015-2017. BAuA, Dortmund

4. Behrens T, Burek K, Pallapies D et al (2019) Decreased psychomotor vigilance of female shift workers after working night shifts. PLoS One 14:e219087

5. Bonnefond A, Harma M, Hakola T et al (2006) Interaction of age with shift-related sleepwakefulness, sleepiness, performance, and social life. Exp Aging Res 32:185-208

6. Brossoit RM, Crain TL, Leslie JJ et al (2019) The effects of sleep on workplace cognitive failure and safety. JOccup Health Psychol 24(4):411-422

7. Chellappa SL, Morris CJ, Scheer F (2018) Daily circadian misalignment impairs human cognitive performance task-dependently. Sci Rep 8:3041

8. Del Rio-Bermudez C, Diaz-Piedra C, Catena A et al (2014) Chronotype-dependent circadian rhythmicity of driving safety. Chronobiol Int 31:532-541

9. Depner CM, Melanson EL, Eckel RH et al (2019) Ad libitum weekend recovery sleep fails to prevent metabolic dysregulation during a repeating pattern of insufficient sleep and weekend recovery sleep.Curr Biol 29:957-967.e954

10. Dijk DJ, Duffy JF, Silva EJ et al (2012) Amplitude reduction and phase shifts of melatonin, cortisol and other circadian rhythms after a gradual advance of sleep and light exposure in humans. PLoSOne 7:e30037

11. Hirschwald B, Heitmann T, Bochmann F et al (2018) Ausgeschlafen ist sicherer! DGUV Forum 2018(11):16-19

12. Horne JA, Ostberg O (1976) A self-assessment questionnaire to determine morningness-eveningness in human circadian rhythms. Int J Chronobiol 4:97-110

13. Osterkamp J (2017) Medizin-Nobelpreis für die innere Uhr. https://www.spektrum.de/ news/medizin-nobelpreis-fuer-die-innere-uhr/ 1507805.Zugegriffen: 3. März 2020

14. Morris DM, Pilcher JJ, Mulvihill JB et al (2017) Performance awareness: predicting cognitive performance during simulated shiftwork using chronobiological measures. Appl Ergon 63:9-16

15. Randler C (2014) CSM. Composite Scale of Morningness - deutsche Fassung. ZPID, Trier https://doi.org/10.23668/psycharchives. 440

16. Randler C (2008) Psychometric properties of the German version of the Composite Scale of Morningness. Biol Rhythm Res 39(2):151-161

17. Randler C, Engelke J (2019) Gender differences in chronotype diminish with age: a metaanalysis based on morningness/chronotype questionnaires. Chronobiol Int 36(7):888-905. https://doi.org/10.1080/07420528.2019.1585867

18. Roenneberg T, Merrow M (2007) Entrainment of the human circadian clock. Cold Spring Harb Symp Quant Biol 72:293-299

19. Roenneberg T, Wirz-Justice A, MerrowM (2003) Life between clocks: daily temporal patterns of human chronotypes. J Biol Rhythms 18:80-90

20. Saksvik IB, Bjorvatn B, Hetland $\mathrm{H}$ et al (2011) Individual differences in tolerance to shift work-a systematic review. Sleep Med Rev 15:221-235

21. Smith CS, Reilly C, Midkiff K (1989) Evaluation of three circadian rhythm questionnaires with suggestions for an improved measure of morningness. J Appl Psychol 74:728-738

22. Stothard ER, McHill AW, Depner CM et al (2017) Circadian entrainment to the natural light-dark cycle across seasons and the weekend. Curr Biol 27(4):508-513

23. Uehli K, Mehta AJ, Miedinger D et al (2014) Sleep problems and work injuries: a systematic review and meta-analysis. Sleep Med Rev 18:61-73
24. Van Dongen HP, Maislin G, Mullington JM et al (2003) The cumulative cost of additional wakefulness: dose-response effects on neurobehavioral functions and sleep physiology from chronic sleep restriction and total sleep deprivation. Sleep 26:117-126 